\title{
ON THE PRODUCT OF VECTOR MEASURES
}

\author{
IGOR KLUVÁNEK
}

(Received 16 August 1970)

Communicated by E. Strzelecki

Let $\mu$ and $\nu$ be measures defined on some $\sigma$-algebras with values in locally convex topological vector spaces $X$ and $Y$, respectively. It is possible [1] to construct their product $\lambda=\mu \times \nu$ as a measure on a $\sigma$-algebra if $\lambda$ is allowed to take its values in $X \otimes_{\varepsilon} Y$, the completion of $X \otimes Y$ in the topology of bi-equicontinuous convergence. The reason is, roughly speaking, that the topology of biequicontinuous convergence on $X \otimes Y$ is coarse enough to make $\lambda \sigma$-additive and the completion $X \otimes_{\varepsilon} Y$ is big enough to accommodate all values of $\lambda$. Here we are going to improve the result by introducing a finer topology on $X \otimes Y$ in which $\lambda$ will be $\sigma$-additive and such that all values of $\lambda$ will belong to the completion of $X \otimes Y$ under that topology. The topology in question is obtained by a slight modification from a topology considered for the first time in the work [3] of Jacobs. Curiously enough, the proof of the improved result is simpler than that of [1] and reduces almost to a direct observation avoiding duality arguments.

Let $X$ and $Y$ be locally convex topological vector spaces. Let the topology of $X$ be given by a family $P$ of semi-norms and that of $Y$ by a family $Q$ of seminorms. For every $u$ in $X \otimes Y$ and for $p \in P, q \in Q$ put

$$
\begin{aligned}
& p \sigma_{l} q(u)=\inf \sup p\left(\sum_{i=1}^{k} \alpha_{i} q\left(y_{i}\right) x_{i}\right) \\
& p \sigma_{r} q(u)=\inf \sup q\left(\sum_{i=1}^{k} \alpha_{i} p\left(x_{i}\right) y_{i}\right),
\end{aligned}
$$

where, in both formulas, the supremum is taken over all choices of numbers $\alpha_{i}$ such that $\left|\alpha_{i}\right| \leqq 1, i=1,2, \cdots, k$, and the infimum is taken over all expression of $u$ in the form

$$
u=\sum_{i=1}^{k} x_{i} \otimes y_{i}
$$

with $x_{i} \in X, y_{i} \in Y, i=1,2, \cdots, k ; k=1,2, \cdots$.

It is readily seen, that $p \sigma_{r} q$ and $p \sigma_{l} q$ are cross-products of seminorms $p$ and $q$. 
The completion of $X \otimes Y$, equipped with the topology generated by the family $\left\{p \sigma_{l} q: p \in P, q \in Q\right\}$ of seminorms, is denoted by $X \otimes_{\sigma l} Y$. Similar meaning has $X \otimes_{\sigma r} Y$. These concepts were introduced by Jacobs in [3]. In fact, he considered only one of the spaces $X \otimes_{\sigma l} Y, X \otimes_{\sigma r} Y$ since it is obvious that $X \otimes_{\sigma l} Y$ is isomorphic to $Y \otimes_{\sigma r} X$. But it is convenient for our purposes to consider them both and we are going to introduce still another one.

For $p \in P$ and $q \in Q$ we define

$$
p \tau q=\frac{1}{2}\left(p \sigma_{l} q+p \sigma_{r} q\right) .
$$

This formula obviously defines a cross-product of semi-norms $p$ and $q$. The completion of $X \otimes Y$ equipped with the topology generated by the family $\{p \tau q: p \in P, q \in Q\}$ will be denoted by $X \otimes_{\mathfrak{\tau}} Y$.

As customary, for $u$ in the form (1) and $p \in P, q \in Q$ we put

$$
p \varepsilon q=\sup \left\{\sum_{i=1}^{k}\left|\left\langle x_{i}, x^{\prime}\right\rangle\left\langle y_{i}, y^{\prime}\right\rangle\right|: x^{\prime} \in U_{p}^{\circ}, y^{\prime} \in U_{q}^{\circ}\right\}
$$

where $U_{p}^{\circ}$ and $U_{q}^{\circ}$ are polars of sets $U_{p}=\{x: p(x) \leqq 1\}$ and $U_{q}=\{y: q(y) \leqq 1\}$, respectively. This is the cross-product of bi-equicontinuous convergence. The correspondencing completed tensor-product space is denoted by $X \in \otimes_{\varepsilon} Y$.

The projective cross-product of $p \in P, q \in Q$ is defined by

$$
\left.p \pi q(u)=\inf \sum_{i=1}^{k} p\left(x_{i}\right) q y_{i}\right)
$$

where the infimum is taken for all expressions of $u$ in the form (1). The completed projective tensor product of $X$ and $Y$ is denoted by $X \otimes_{\pi} Y$.

It is readily seen that, for any $p \in P$ and $q \in Q$,

$$
p \varepsilon q \leqq{ }_{p \sigma_{r} q}^{p \sigma_{\imath} q} \leqq p \tau q \leqq p \pi q
$$

on $X \otimes Y$. It follows that the identity map on $X \otimes Y$ extends uniquely to continuous inclusions

$$
X \otimes_{\pi} Y \rightarrow X \otimes_{\tau} Y \searrow_{\searrow}^{\nearrow} X \otimes_{\sigma r} Y \otimes_{\sigma l} Y \otimes_{\varepsilon} Y .
$$

Moreover, examples can be exhibited showing that all indicated inclusions could be strict.

Let $S$ and $T$ be abstract sets. Let $\mathscr{S}$ be a ring (possibly $\sigma$-ring, $\sigma$-algebra, etc.) of subsets of $S$ and $\mathscr{T}$ a ring of subsets of $T$. We denote

$$
\mathscr{S} \times \mathscr{T}=\{A \times B: A \in \mathscr{S}, B \in \mathscr{T}\} .
$$


Further, $\mathscr{S} \otimes \mathscr{T}$ will stand for the ring generated by $\mathscr{S} \times \mathscr{T}, \mathscr{S} \otimes_{\sigma} \mathscr{T}$ will denote the $\sigma$-ring generated by $\mathscr{S} \times \mathscr{T}$ and, finally, the symbol $\mathscr{S} \otimes_{\delta} \mathscr{T}$ will be used for the $\delta$-ring generated by $\mathscr{S} \times \mathscr{T}$.

The term measure or vector measure will mean a $\sigma$-additive measure, i.e. a $\sigma$-additive map from a ring of sets into a topological vector space.

THEOREM. Let $\mathscr{S}$ and $\mathscr{T}$ be $\sigma$-rings and $\mu: \mathscr{S} \rightarrow X$ and $v: \mathscr{T} \rightarrow Y$ vector measures. Then there exists a vector measure $\lambda: \mathscr{S} \otimes_{\sigma} \mathscr{T} \rightarrow X \otimes_{\tau} Y$ such that

$$
\lambda(A \times B)=\mu(A) \otimes v(B), A \in \mathscr{S}, B \in \mathscr{T} .
$$

Proof. Let $p \in P$ and $q \in Q$. Let $m$ be a real finite non-negative measure on $\mathscr{S}$ such that $m(A) \rightarrow 0, A \in \mathscr{S}$, implies $p(\mu(A)) \rightarrow 0$ and let $n$ be a finite non-negative measure on $\mathscr{T}$ such that $n(B) \rightarrow 0, B \in \mathscr{T}$, implies $q(v(B)) \rightarrow 0$ The existence of such measures $m$ and $n$ follows e.g. from Theorem 4.2 in [4]. The same Theorem gives that it it now enough to prove that $m \times n(E) \rightarrow 0$, $E \in \mathscr{S} \otimes \mathscr{T}$, implies $p \tau q(\lambda(E)) \rightarrow 0$, where $\lambda$ is the unique $X \otimes Y$-valued additive function on $\mathscr{S} \otimes \mathscr{T}$ satisfying (2). We are going to prove it. But it means that we are going to prove that $p \sigma_{l} q(\lambda(E)) \rightarrow 0$ and $p \sigma_{r} q(\lambda(E)) \rightarrow 0$ if $m \times n(E) \rightarrow 0$. Because of symmetry we will prove only the statement concerning $\sigma_{l}$.

It is known that, there is $A_{0} \in \mathscr{S}$ and $B_{0} \in \mathscr{T}$ such that $m(A)=m\left(A \cap A_{0}\right)$, for $A \in \mathscr{S}$, and $n(B)=n\left(B \cap B_{0}\right)$, for $B \in \mathscr{T}$. So, without loss of generality we can assume that $m(S)=1, n(T)=1$ and that

$$
p\left(\sum_{i=1}^{k} \alpha_{i} \mu\left(A_{i}\right)\right) \leqq 1, \quad q(v(B)) \leqq 1
$$

for all collections $A_{1}, A_{2}, \cdots, A_{k}$ of pairwise disjoint sets in $\mathscr{S}$, all numbers $\alpha_{i}$ with $|\alpha| \leqq 1, i=1,2, \cdots, k$, and all $B \in \mathscr{T}$.

Let $\varepsilon>0$. Let $\delta_{1}>0$ be such that $m(A)<\delta_{1}, A \in \mathscr{S}$, implies $p(\mu(A))<\varepsilon / 8$. Let $\delta_{2}>0$ be such that $n(B)<\delta_{2}, B \in \mathscr{T}$, implies $q(v(B))<\varepsilon / 2$. Let $\delta=\delta_{1} \delta_{2}$ Let $E \in \mathscr{S} \otimes \mathscr{T}, m \times n(E)<\delta$. Suppose $E$ to be expressed in the form

$$
E=\bigcup_{i=1}^{k} A_{i} \times B_{i}, A_{i} \in \mathscr{S}, B_{i} \in \mathscr{T},
$$

with pairwise disjoint $A_{i}, i=1,2, \cdots, k$. Assume the notation arranged in such a way that $n\left(B_{i}\right)<\delta_{2}$, for $1 \leqq i \leqq k$, and $n\left(B_{i}\right) \geqq \delta_{2}$, for $l+1 \leqq i \leqq k$, where $0 \leqq l \leqq k$. Since $m \times n(E)<\delta_{1} \delta_{2}$,

$$
m\left(A_{l+1} \cup \cdots \cup A_{k}\right)<\delta_{1} .
$$

By definition of $p \sigma_{l} q$ we have (the supremum is taken everywhere over all choices of numbers $\alpha_{i}$ with $\left|\alpha_{i}\right| \leqq 1$ ): 


$$
\begin{aligned}
p \sigma_{l} q(\lambda(E)) & \leqq \sup p\left(\sum_{i=1}^{k} \alpha_{i} q\left(v\left(B_{i}\right)\right) \mu\left(A_{i}\right)\right) \\
& \leqq \sup p\left(\sum_{i=1}^{l} \alpha_{i} q\left(v\left(B_{i}\right)\right) \mu\left(A_{i}\right)\right)+\sup p\left(\sum_{i=l+1}^{k} \alpha_{i} q\left(v\left(B_{i}\right)\right) \mu\left(A_{i}\right)\right) \\
& \leqq \frac{1}{2} \varepsilon \sup p\left(\sum_{i=1}^{l} \frac{2}{\varepsilon} \alpha_{i} q\left(v\left(B_{i}\right)\right) \mu\left(A_{i}\right)\right)+\sup p\left(\sum_{i=l+1}^{k} \alpha_{i} \mu\left(A_{i}\right)\right) \\
& \leqq \frac{1}{2} \varepsilon+4 \cdot \frac{1}{8} \varepsilon=\varepsilon
\end{aligned}
$$

since

$$
\begin{array}{r}
\left|\frac{2}{\varepsilon} \alpha_{i} q\left(v\left(B_{i}\right)\right)\right| \leqq 1, i=1,2, \cdots, l ;\left|\alpha_{i} q\left(v\left(B_{i}\right)\right)\right| \leqq 1, \\
i=l+1, \cdots, k .
\end{array}
$$

CoRollary. 1. If $\mathscr{S}$ and $\mathscr{T}$ are $\sigma$-rings and $\mu: \mathscr{S} \rightarrow X$ and $v: \mathscr{T} \rightarrow X$ are vector measures, then there exists a vector measure $\lambda: \mathscr{S} \otimes_{\sigma} \mathscr{T} \rightarrow X \otimes_{\varepsilon} Y$ such that (2) holds.

ProOF. $X \otimes_{\tau} Y$ is continuously included in $X \otimes_{\varepsilon} Y$.

Obviously in the Corollary the space $X \otimes_{\varepsilon} Y$ can be replaced by the completion $X \otimes_{\eta} Y$ of $X \otimes Y$ with respect to any cross-product topology $\eta$ coarser than $\tau$ and finer than $\varepsilon$.

If $\mathscr{P}$ is a $\sigma$-algebra, then $c a(\mathscr{S})$ denotes the Banach space of all complex measures on $\mathscr{S}$ with the variation on the whole space serving as a norm.

CoRollaRy 2. (Cf. [1], Lemma.) Let $\mathscr{S}$ and $\mathscr{T}$ be $\sigma$-algebras. Let $M \subset \operatorname{ca}(\mathscr{S})$ $N \subset c a(\mathscr{T})$ be relatively weakly compact sets. Then $\{m \times n: m \in M, n \in N\}$ is a relatively weakly compact subset of ca( $\left.\mathscr{S} \otimes_{\sigma} \mathscr{T}\right)$.

Proof. Let $l^{\infty}(M)$ be the Banach space of all bounded functions $x$ on $M$ written as vectors $x=\left(x_{m}\right)_{m \in M}$ equipped with sup-norm. By Theorem IV.9.1 in [2], $M$ is relatively weakly compact if and only if $M$ is bnunded and the $\sigma$-additivity is uniform in $M$. Hence, if, for every $A \in \mathscr{S}$, we define $\mu(A)=\left(x_{m}\right)_{m \in M}$ where $x_{m}=m(A), m \in M$, then $A \rightarrow \mu(A), A \in \mathscr{S}$, we define measure on $\mathscr{S}$ with values in $l^{\infty}(M)$. Similarly we define $v: \mathscr{T} \rightarrow l^{\infty}(N)$. By Corollary 1 there is a vector measure $\lambda: \mathscr{S} \otimes_{\sigma} \mathscr{T} \rightarrow l^{\infty}(M) \otimes_{\varepsilon} l^{\infty}(N)$ satisfying (2). By Corollary IV.10.2 in [2], the set of measure $\left\{z^{\prime} \circ \lambda: z^{\prime} \in U^{\prime}\right\}$, where $U^{\prime}$ is the unit ball in the dual-space to $l^{\infty}(M) \otimes_{\varepsilon} l^{\infty}(N)$, is relatively weakly compact in ca $\left(\mathscr{S} \otimes_{\varepsilon} \mathscr{T}\right)$. So it suffices to show that

$$
\{m \times n: m \in M, n \in N\} \subset\left\{z^{\prime} \circ \lambda: z^{\prime} \in U^{\prime}\right\} .
$$


But $m \times n=z^{\prime} \circ \lambda$ if $z^{\prime}$ is the continuous linear functional on $l^{\infty}(M) \otimes_{\varepsilon} l^{\infty}(N)$ for which $z^{\prime}(x \otimes y)=x_{m} y_{n}, x \in l^{\infty}(M), y \in l^{\infty}(N)$. By the definition of the $\varepsilon$ product so defined $z^{\prime}$ belongs to $U^{\prime}$.

COROLlary 3. Let $\mathscr{S}$ and $\mathscr{T}$ be $\delta$-rings and $\mu: \mathscr{S} \mathscr{T} \rightarrow Y$ vector measures. Then there is a vector measure $\lambda: \mathscr{S} \otimes_{\delta} \mathscr{T} \rightarrow X \otimes Y$. satisfying (2).

Proof. Starting from $\lambda: \mathscr{S} \otimes \mathscr{T} \rightarrow X \otimes Y$ defined by the requirement (2) and that of additivity we have to show that $\lambda$ can be extended as a $\sigma$-additive function onto whole of $\mathscr{S} \otimes_{\delta} \mathscr{T}$ taking values in $X \otimes_{\tau} Y$. To achieve this it is sufficient to show that if $E_{j} \in \mathscr{S} \otimes \mathscr{T}, E_{j} \supset E_{j+1}, j=1,2, \cdots$, then $\lim _{j} \lambda\left(E_{j}\right)$ exists in $X \otimes_{\tau} Y$ and if it happens that $\bigcap_{j=1}^{\infty} E_{j}=Q$ then this limit is 0 . See theorem in [5]). Since there is $A_{0} \in \mathscr{S}$ and $B_{0} \in \mathscr{T}$ such that $E \subset A_{0} \times B_{0}$ the result will follow from Theorem by applying it to the restriction of $\mu$ to the system $\left\{A: A \in \mathscr{S}, A \subset A_{0}\right\}$ and to the restriction of $v$ to the system $\left\{B: B \in \mathscr{T}, B \subset B_{0}\right\}$. These systems are $\sigma$-algebras of subsets of $A_{0}$ and $B_{0}$ respectively.

\section{References}

[1] M. Duchoň and I. Kluvánek. 'Inductive tensor product of vector-valued measures', Matematický Časopis 17 (1967), 108-111.

[2] N. Dunford and J. T. Schwartz, Linear Operators I (New York, 1958).

[3] H. Jacobs, Ordered topological tensor products (Thesis, University of Illinois, Urbana 1969).

[4] I. Kluvánek, 'Contribution to the theory of vector measures' (Russian), Matematiko-fyzikálny časopis 11 (1961), 173-191.

[5] I. Kluvánek, 'Contribution to the theory of vector measures II' (Russian), Matematickofyzikálny časopis 16 (1966), 76-81.

University of Illinois, Urbana, Illinois, U.S.A.

Present address

The Flinders University of South Australia 